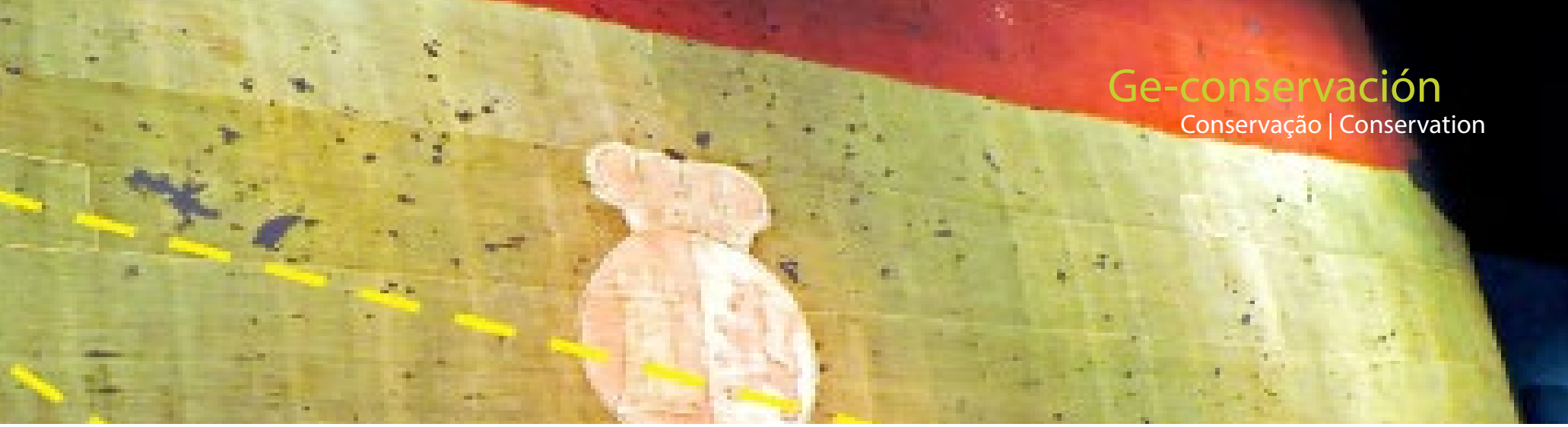

\title{
Propuesta de protocolo de valoración de la acidez ambiental en salas y vitrinas de la exposición temporal El último viaje de la fragata Mercedes. La razón frente al expolio (Museo Naval, Madrid)
}

\author{
Javier Peña Poza, José María Gálvez Farfán, Mercedes González Rodrigo, Susana García Ramírez, María \\ Ángeles Villegas Broncano, Manuel García Heras
}

\begin{abstract}
Resumen: En este trabajo se ha realizado la valoración ambiental, tanto en salas como en el interior de vitrinas, en la exposición temporal El último viaje de la fragata Mercedes. La razón frente al expolio, celebrada en el Museo Naval de Madrid. El estudio se realizó mediante un protocolo de seguimiento de la acidez ambiental que utiliza sensores ópticos. Los resultados obtenidos demostraron que los valores promedio de $\mathrm{pH}$ se situaban cercanos a la neutralidad teórica (riesgo de choque ácido bajo), excepto en algunas vitrinas en las que se detectaron valores ligeramente ácidos. Los datos indicaron que los sistemas de ventilación y los materiales empleados en salas y vitrinas (p. ej. pinturas, barnices, adhesivos, etc.) eran adecuados ya que no provocaron valores de $\mathrm{pH}$ perjudiciales para la correcta conservación de las colecciones.
\end{abstract}

Palabras clave: conservación preventiva, sensor, acidez ambiental, exposición temporal, Museo Naval

Proposed protocol for assessing environmental acidity in exhibition rooms and showcases of the temporary exhibition The last trip of the Mercedes frigate. The reason against the expolio (The Navy Museum, Madrid)

\begin{abstract}
In this work an environmental valuation, either in exhibition rooms or inside showcases, has been undertaken in the temporary exhibition The last trip of the Mercedes frigate. The reason against the expolio, held in the Navy Museum of Madrid. The study was accomplished through a protocol for monitoring environmental acidity which uses optical sensors. The results obtained demonstrated that average values of $\mathrm{pH}$ were located near the theoretical neutrality (low risk of acid shock), except in some showcases in which slightly acid values were detected. The data indicated that ventilation systems and materials employed in exhibition rooms and showcases (e.g. paints, varnishes, adhesives, etc.) were adequate since they did not cause dangerous $\mathrm{pH}$ values for the proper conservation of collections.
\end{abstract}

Key words: preventive conservation, sensor, environmental acidity, temporary exhibition, Navy Museum

\section{Proposta de protocolo de avaliação da acidez ambiental em salas e montras da exposição tem- porária A última viagem da fragata Mercedes. A razão frente ao espolio ( Museu Naval de Madrid)}

Resumo: Neste trabalho efectuou-se a avaliação ambiental, tanto em relação às salas como em relação ao interior das montras, da exposição temporária A última viagem da fragata Mercedes. A razão frente ao espolio, realizada no Museu Naval de Madrid. O estudo realizou-se mediante um protocolo de seguimento da acidez ambiental que utiliza sensores ópticos. Os resultados obtidos demonstraram que os valores médios de $\mathrm{pH}$ se situavam perto da neutralidade teórica ( risco leve de choque ácido), excepto em algumas montras em que foram detectados valores ligeiramente ácidos. Os dados indicaram que os sistemas de ventilação e os materiais utilizados nas salas e nas montras ( por ex: tintas, vernizes, colas, etc) foram os adequados visto não terem causado valores de pH prejudiciais para a conservação correta das colecções. 


\section{Introducción}

Para la conservación preventiva de las colecciones albergadas en un museo, estén éstas expuestas o almacenadas en sus fondos, la evaluación y control periódicos de las condiciones microclimáticas y la presencia de posibles contaminantes, tanto en las distintas salas como en el interior de vitrinas y otros tipos de contenedores, son actividades que requieren un trabajo sistemático a corto, medio y largo plazo (Michalski 1998; Thomson 2008). La conservación preventiva es una estrategia que no requiere ninguna intervención directa en las colecciones o en los objetos que las componen, en contraposición a la conservación curativa, en la que se interviene de forma directa en la colección o en los objetos para evitar riesgos de deterioro importantes o, a veces, irreversibles.

Solo en las últimas décadas la conservación preventiva ha experimentado un impulso notable entre las principales tareas de las instituciones museológicas. Este impulso se ha realizado desde organismos como el Consejo Internacional de Museos (ICOM) o el Centro para el Estudio de la Conservación y Restauración de los Bienes Culturales (ICCROM) que, en varias reuniones como la celebrada en Vantaa (Finlandia) en el año 2000 (VV.AA. 2000), han sentado las bases de una estrategia común europea en temas como la conservación preventiva en museos y, en especial, sobre calidad del aire y presencia de contaminantes en su interior (Camuffo et al. 2001; Muñoz-Campos 2006; Forniés Matías 2011, 2012; García Fernández 2014: 11-13). Estas bases son las que ha recogido el vigente primer Plan Nacional de Conservación Preventiva en España, aprobado en 2011 por el entonces Ministerio de Cultura (PNCP 2011).

Una actividad que cada vez tiene un mayor protagonismo en los museos es la realización de exposiciones temporales. Las opiniones de los especialistas sobre los beneficios de estas exposiciones son casi tan numerosas como las que señalan sus efectos adversos. Entre los argumentos esgrimidos para subrayar su conveniencia destacan aquéllos que inciden en su capacidad para renovar y aumentar los visitantes del museo, así como en las posibilidades que ofrecen para plantear nuevas experiencias expositivas. Por el contrario, entre los argumentos que se utilizan para incidir en sus efectos adversos destacan los que hacen referencia al cambio de ubicación de los objetos que conlleva, entre otros efectos, alteraciones ambientales y procesos de manipulación y transporte, con todo lo que la movilidad representa en cuanto a riesgos de golpes, vibraciones y posible rotura se refiere (VV.AA. 2006; Fernández 2008).

En un proyecto de investigación que se está llevando a cabo actualmente (MINECO Ref. HAR2012-30769), se ha desarrollado un protocolo de seguimiento y control de la acidez ambiental en espacios museísticos para identificar, evaluar, controlar y minimizar posibles riesgos de deterioro debidos a choque ácido. Esta metodología, que emplea sensores químicos de respuesta óptica, ha resultado eficaz en distintos museos en los que se ha aplicado y ensayado
(Peña-Poza et al. 2011 a; Llorente et al. 2013), incluyendo la colección permanente y otros espacios del Museo Naval de Madrid (Peña-Poza et al. 2014). El nuevo protocolo es sencillo, tanto en la obtención de las medidas como en el procesado de los datos, y podría llevarse a cabo por los profesionales de un museo tras un corto periodo de aprendizaje y entrenamiento.

\section{Objetivos}

Con motivo de la celebración en el Museo Naval de Madrid de la exposición temporal El último viaje de la fragata Mercedes. La razón frente al expolio, entre junio de 2014 y enero de 2015, se planteó un estudio de valoración ambiental de esta exposición a través del protocolo de seguimiento y control de la acidez ambiental mencionado anteriormente. El objetivo principal de este estudio consistió en evaluar, controlar y minimizar posibles riesgos de choque ácido en dicha exposición temporal para determinar si los factores "positivos y beneficiosos" podrían servir para contrarrestar los "negativos y perniciosos" derivados del cambio de ubicación de las piezas que componen la exposición. Aunque como ya se ha indicado se han realizado valoraciones ambientales en la colección permanente (Peña-Poza et al. 2014), un estudio de conservación preventiva de estas características es la primera vez que se ha llevado a cabo en una exposición temporal. Dicho estudio se centró en la determinación de valores de $\mathrm{pH}$ ambiental en las distintas salas de la muestra temporal. Aparte de los valores de $\mathrm{pH}$ obtenidos con el protocolo, el museo registró los parámetros habituales de estabilidad microclimática como humedad relativa, temperatura, incidencia lumínica o radiación UV e infrarroja. Además, en las salas de exposición temporal se mantienen constantes las condiciones ambientales de humedad relativa $y$ temperatura, con intervalos de variación no superiores a 5 $\%$ y a $2^{\circ} \mathrm{C}$, respectivamente, por lo que se considera que la variación de estos parámetros es despreciable respecto a la obtención de los valores de $\mathrm{pH}$.

El protocolo de seguimiento y control de la acidez ambiental mediante el uso de sensores químicos de respuesta óptica presenta ventajas importantes frente a otros sistemas de medida como las tiras de papel de $\mathrm{pH}$ o el test de Oddy (Oddy 1973) y sus variantes (Bamberger et al. 1999). El papel $\mathrm{pH}$ es un papel impregnado con sustancias químicas sensibles a los cambios de acidez o basicidad. Cambian de color cuando se sumergen en disoluciones acuosas o líquidos, en general, y después de 10-30 segundos adquieren un determinado color que indica cualitativamente el pH por comparación con una escala de color. Las medidas con papel $\mathrm{pH}$ dependen de la subjetividad visual del observador $y$, por lo tanto, no son exactas. El uso del papel $\mathrm{pH}$ previamente humedecido con agua y/o con cualquier otra sustancia, incluso estable al aire, para evaluar el pH del aire no es adecuado ni recomendable. Esto es debido a que en las variaciones de color del papel se producen interferencias procedentes del propio $\mathrm{pH}$ de 
las sustancias humectantes, así como las producidas por la descomposición química de las sustancias sensibles que impregnan el papel. En otras palabras, la calidad del diagnóstico del $\mathrm{pH}$ ambiental utilizando papel $\mathrm{pH}$ es imprecisa, inexacta y con una probabilidad muy elevada de error, ya que se utilizaría en unas condiciones distintas y para una finalidad distinta de las que están previstas para el papel pH. El uso de los sensores ópticos de pH ambiental basados en la tecnología Sol-gel utilizados en el protocolo mencionado, permite la determinación cuantitativa del pH en el aire y en cualquier fase gaseosa con una precisión de $\pm 0,1$ unidades de la escala de $\mathrm{pH}$. Por lo tanto, es un procedimiento de evaluación que garantiza la calidad del diagnóstico sobre el $\mathrm{pH}$ del aire, y elimina las fuentes de error debidas a la manipulación de líquidos, a la agudeza visual del observador y a la descomposición de los reactivos sensibles del papel $\mathrm{pH}$.

Respecto al test de Oddy, el control de materiales aptos para la conservación, es decir, materiales que no emitan sustancias ácidas con el tiempo (Gall Ortlik 2004), puede realizarse directamente con uno de estos sensores sin necesidad de utilizar chapas testigo de metales como plata, cobre o plomo y sin necesidad de esperar los 28 días que el test estipula para obtener resultados, que se basan en los mecanismos de corrosión de estos metales por la presencia de especies ácidas en un ambiente húmedo dentro del recipiente hermético en el que se lleva a cabo. Con estos sensores, las respuestas de acidez ambiental se obtienen de forma limpia en menos de 24 horas y se pueden repetir las veces necesarias dentro del periodo de medida que se estipule. Además, la medida es cuantitativa y no cualitativa como ocurre con las chapas de los metales mencionados, en los que la acidez se deduce a partir de la corrosión y/o patinación que puede presentar su superficie.

\section{Metodología}

\section{Preparación de los sensores}

Para la valoración ambiental realizada en este trabajo se prepararon y utilizaron sensores de acidez basados en la tecnología Sol-gel (Peña Poza 2014: 18). Los sustratos o soportes de los sensores fueron láminas de vidrio común (portas de microscopio) en los que se depositó un recubrimiento delgado (300-400 nm) por la técnica de inmersiónextracción.

El sol o suspensión coloidal que da lugar al recubrimiento sensible de los sensores se preparóa partir de una disolución de $2,96.10^{-4}$ moles de rojo de clorofenol como fase sensora disuelto en 0,18 moles de etanol $y$, por otra parte, de una suspensión de $0,46.10^{-1}$ moles de tetraetóxido de silicio diluido con 0,18 moles de etanol y 0,04 moles de ácido clorhídrico concentrado al 37 \% en peso. La disolución de rojo de clorofenol y la suspensión de tetraetóxido de silicio se adicionan en un vaso de precipitados donde se agitan para su homogenización.
Para la deposición de los recubrimientos en los sustratos de vidrio por inmersión-extracción se utilizó un dispositivo que permite introducir los sustratos de vidrio en vertical y lentamente en el vaso de precipitados que contiene el sol indicado anteriormente. Una vez que el sustrato queda mojado por el sol se extrae a una velocidad controlada de $1,35 \mathrm{~mm} . \mathrm{s}^{-1}$ y se depositan en un soporte refractario que se introduce en una estufa de laboratorio de aire forzado estabilizada a $60^{\circ} \mathrm{C}$. Los sustratos recubiertos (sensores) permanecen en la estufa durante $72 \mathrm{~h}$, lo que garantiza su secado y densificación parcial. De este modo los sensores pueden manipularse fácilmente, además de conservar su actividad o sensibilidad óptica cuando se exponen a cualquier ambiente.

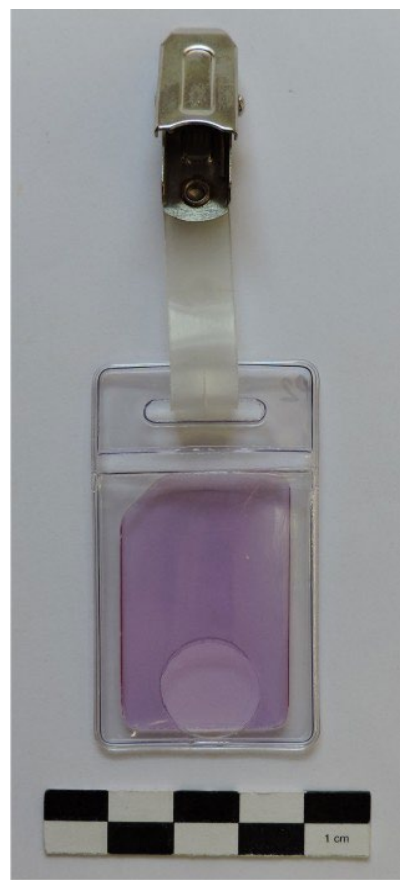

Figura 1.- Sensor de acidez dentro de una funda protectora provista de pinza de sujeción

Posteriormente, los sensores se acondicionan eliminando por corte y pulido las partes del porta de vidrio que no han quedado recubiertas con el sol o que presentan los defectos característicos del borde o nivel del sol durante la deposición por inmersión-extracción. La figura 1 muestra el aspecto de un sensor una vez acondicionado y colocado dentro de una funda protectora de material polimérico que se utilizó para su instalación en el Museo Naval.

Como se puede observar en la figura 1, las fundas protectoras disponen de una perforación de forma circular por cada cara que facilita el contacto directo del ambiente (aire) con la superficie sensible del sensor.

\section{Validación y calibración de los sensores}

Antes de utilizar los sensores es necesario llevar a cabo un estudio de su respuesta óptica: absorción visible 
neta, reproducibilidad, reversibilidad, capacidad de regeneración, etc. Por esta razón se desarrolló un protocolo de calidad o validación de los sensores que consistió en registrar su absorción visible (color) a pH 5,0 y 7,0 para la longitud de onda de la banda principal de absorción del rojo de clorofenol, que se sitúa a 575 $\mathrm{nm}$. La absorción visible de los sensores se registró con un espectrofotómetro Ocean Optics modelo HR-4000. Los sensores cuya absorción visible se desvían más de 0,2 unidades equivalentes de $\mathrm{pH}$ respecto al $\mathrm{pH}$ que corresponde al promedio calculado de intensidad de absorción en $575 \mathrm{~nm}$, se desechan. También se descartan aquellos sensores cuya absorción visible se desvía notablemente del valor calculado de desviación típica obtenida para las medidas registradas a un mismo $\mathrm{pH}$.

Tras la validación, los sensores se calibraron. En esta operación se utilizaron los sensores validados cuya absorción visible se corresponde con los valores menores de desviación del pH promedio y de desviación típica entre sus medidas. Para cada sensor de los seleccionados para la calibración se obtienen 5 valores de absorción a $575 \mathrm{~nm}$, para distintos valores de $\mathrm{pH}$ comprendidos entre 5,0 y 8,0 . Para ello se utilizaron disoluciones tampón de $\mathrm{pH} 5,0 ; 6,0 ; 7,0$ y 8,0 . Una vez registradas las absorciones de cada sensor se calculan los valores promedio. Cada valor promedio de absorción se considera el valor característico para cada pH entre 5,0 y 8,0. La gráfica de calibración se elabora a partir de las ecuaciones de las rectas consecutivas que unen los valores característicos mencionados. Los sensores alcanzan una precisión de $\pm 0,15$ de la escala de $\mathrm{pH}$, y su tiempo mínimo de respuesta repetible en fase gaseosa (por ejemplo, en aire) se estima en $24 \mathrm{~h}$.

\section{Instalación de los sensores y registro de datos}

Los sensores de acidez se instalaron en las posiciones que se indican en la tabla 1. El la figura 2 se pueden localizar las posiciones sobre el plano de las distintas salas que componen la exposición temporal.

Las respuestas ópticas de los sensores (absorción visible o color) se tomaron al cabo de 24 h y cada 24 h en días sucesivos. En algunas ocasiones los registros se efectuaron cada 48 o 72 h. La evaluación ambiental completa se realizó a lo largo de 8 días.

Los resultados se registraron con una unidad de medida portátil fabricada según una patente de invención de algunos de los presentes autores (Villegas Broncano et

\begin{tabular}{|c|c|c|}
\hline Posición & Sala & Descripción \\
\hline 1 & Sala 7 & En ambiente de sala sobre la reproducción del puente de artillería de la fragata \\
\hline 2 & Sala 6 & En ambiente de sala en el interior del modelo de la fragata \\
\hline 3 & Sala 6 & En ambiente de sala colgado en un extremo de la bandera \\
\hline 4 & Sala 4 & En ambiente de sala del audiovisual sobre el combate en Cabo Sta. María \\
\hline 5 & Entrada & En ambiente de sala en el pasillo de entrada \\
\hline 6 & Sala 1 & En ambiente de sala colgado del cuadro del retrato de Godoy \\
\hline 7 & Sala 2 & En el interior de vitrina con Reales Ordenanzas de Domigo de Grandallana \\
\hline 8 & Sala 3 & En ambiente de sala sobre un maniquí en la escenografía de la mesa de navegación \\
\hline 9 & Sala 4 & En ambiente de sala del audiovisual sobre el combate en Cabo Sta. María \\
\hline 10 & Sala 6 & En ambiente de sala en el interior del modelo de la fragata \\
\hline 11 & Sala 6 & En ambiente de sala colgado en un extremo de la bandera \\
\hline 12 & Sala 6 & En el interior de vitrina con reglamento de maderas para la construcción de barcos \\
\hline 13 & Sala 6 & En el interior de vitrina con objetos de metal y madera \\
\hline 14 & Sala 6 & En el interior de vitrina con una coracha que contiene quinina \\
\hline 15 & Sala 6 & En el interior de vitrina con monedas de plata y de oro \\
\hline 16 & Sala 6 & En el interior de vitrina con maroma y otros objetos \\
\hline 17 & Sala 7 & En ambiente de sala sobre la reproducción del puente de artillería de la fragata \\
\hline 18 & Sala 8 & En ambiente de sala del audiovisual sobre El expolio \\
\hline 19 & Sala 6 & En interior de vitrina con una vasija de cerámica y naipes \\
\hline 20 & Sala 6 & En ambiente de sala junto a un barril de roble \\
\hline
\end{tabular}

Tabla 1.- Posiciones de instalación de los sensores de acidez 


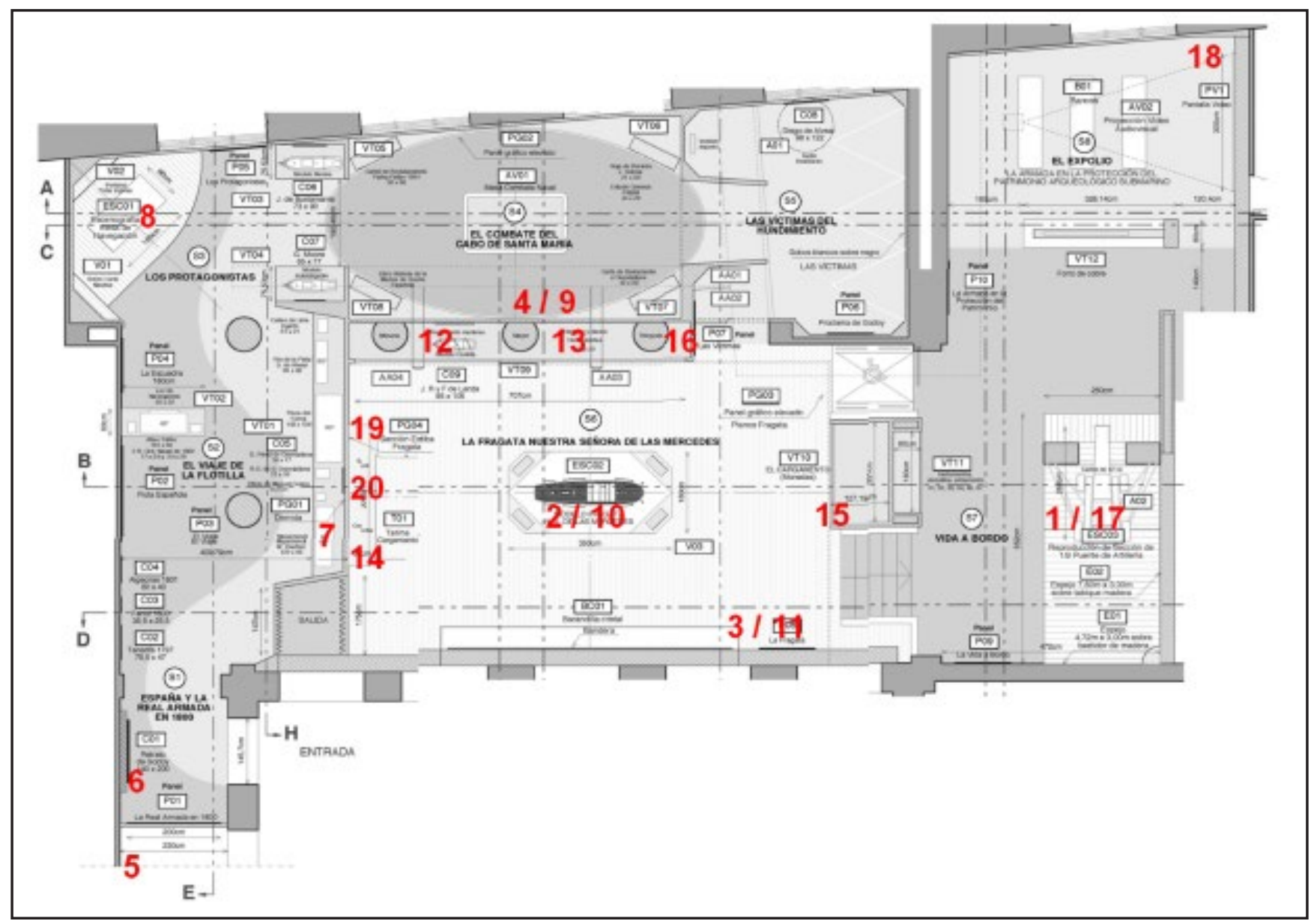

Figura 2.- Plano de las salas de la exposición temporal con las posiciones de instalación de los sensores de acidez descritas en la tabla 1

al. 2010). Esta unidad de medida basa su funcionamiento en la generación de una señal eléctrica proporcional a la absorción visible (color) del sensor. Dispone de un sistema de calibración de la señal eléctrica en función de la temperatura, de modo que la unidad se puede usar tanto en interiores como en exteriores sin que haya interferencias debidas a los cambios bruscos de temperatura. Además, la unidad de medida está provista de un sensor de humedad relativa externo al cuerpo de dicha unidad, lo que permite relacionar las medidas de $\mathrm{pH}$ con el valor de humedad relativa de la zona a evaluar en el momento del registro de los resultados. La comunicación de la unidad con un PC se realiza mediante un cable USB, que también permite la alimentación de la misma. La unidad de medida está asociada a un software de adquisición, registro y tratamiento de los resultados, también desarrollado en la patente mencionada (Villegas Broncano et al. 2010).

Se llevaron a cabo dos evaluaciones ambientales, la primera en julio de 2014, como una primera aproximación, instalando los sensores exclusivamente en posiciones de ambiente de sala [tabla 1 y figura 2, posiciones 1 a 4]; y la segunda en octubre de 2014, en la que los sensores se instalaron tanto en ambiente de sala como en el interior de vitrinas [tabla 1 y figura 2, posiciones 5 a 20]. Los resultados obtenidos en ambas evaluaciones ambientales permitían, en principio, establecer comparaciones entre los resultados característicos de una temporada cálida y una temporada fría.

\section{Resultados y discusión}

La figura 3 recoge los valores promedio de $\mathrm{pH}$ registrados en las posiciones seleccionadas siguiendo criterios de conservación preventiva para la instalación de los sensores.

Todos los valores promedio se encuentran en el intervalo de $\mathrm{pH}$ 6,54-7,28, esencialmente neutro y, por lo tanto, adecuado en términos de conservación preventiva de bienes culturales. En general se considera que un $\mathrm{pH}$ ambiental en el intervalo 6,5-7,5 es correcto para la conservación, y que las variaciones dentro de dicho intervalo no son perjudiciales ni suponen peligro de degradación o corrosión para los materiales de los bienes culturales (Peña Poza 2014: 83).

En los resultados promedio de la figura 3 se aprecia una tendencia diferente entre los resultados procedentes de los sensores instalados en el ambiente de sala y los resultados 


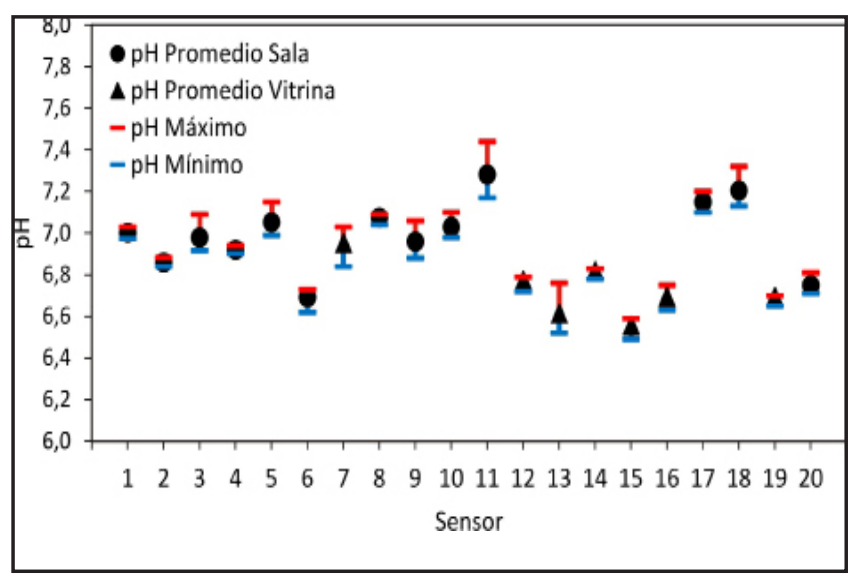

Figura 3.- Valores promedio de $\mathrm{pH}$ ambiental detectado por los sensores instalados en las diversas posiciones de la exposición temporal

procedentes de los sensores instalados en el interior de vitrinas. En general los resultados de $\mathrm{pH}$ obtenidos en el interior de vitrinas son menores (más ácidos) que los registrados en el ambiente de sala. Esta tendencia se puede explicar por las diferencias de volúmenes relativos (en sala y en el interior de una determinada vitrina) y por el número neto de objetos ubicados en cada caso, ya que en las vitrinas dicho número de objetos por unidad de volumen de aire es siempre mayor que en las salas. Este hecho contribuye de forma notable a la generación de ambientes locales o microambientes que probablemente se enriquecen de emisiones, no siempre neutras desde el punto de vista del $\mathrm{pH}$ ambiental, procedentes de los materiales con los que están construidas las vitrinas o bien de los propios bienes culturales. El resultado puede ser una acumulación de especies químicas volátiles y no volátiles (por ejemplo, ácidos orgánicos como el acético o fórmico, y aldehídos u otras especies de características ácidas) que contribuyen a disminuir el pH hasta valores ácidos o casi ácidos. A este respecto obsérvese en la figura 3 los resultados promedio de las posiciones $7,12,13,14,15,16$ y 19, correspondientes al interior de vitrinas, cuyos datos de $\mathrm{pH}$ promedio son de los más bajos (más ácidos) registrados.

En cuanto a los resultados de $\mathrm{pH}$ absoluto registrados [tabla 2, columnas "máximo" y "mínimo"], se observa que el valor máximo de $\mathrm{pH}(7,44)$ corresponde a la posición 11 [tabla 1, ambiente de sala, junto a una bandera de grandes dimensiones], y que el valor mínimo de $\mathrm{pH}(6,49)$ corresponde a la posición 15 [tabla 1, interior de la vitrina que contiene fundamentalmente monedas de plata y de oro rescatadas del fondo marino en donde se encontraba el pecio]. Aunque ambos valores de $\mathrm{pH}(7,44$ y 6,49$)$ se

\begin{tabular}{|c|c|c|c|c|}
\hline Posición & Tipo instalación & Periodo exposición & pH máximo & pH mínimo \\
\hline 1 & Sala & \multirow{4}{*}{ Julio 2014} & 7,03 & 6,97 \\
\hline 2 & Sala & & 6,88 & 6,84 \\
\hline 3 & Sala & & 7,09 & 6,92 \\
\hline 4 & Sala & & 6,94 & 6,90 \\
\hline 5 & Sala & \multirow{16}{*}{ Octubre 2014} & 7,15 & 6,99 \\
\hline 6 & Sala & & 6,73 & 6,62 \\
\hline 7 & Vitrina & & 7,03 & 6,84 \\
\hline 8 & Sala & & 7,09 & 7,04 \\
\hline 9 & Sala & & 7,06 & 6,88 \\
\hline 10 & Sala & & 7,10 & 6,98 \\
\hline 11 & Sala & & 7,44 & 7,17 \\
\hline 12 & Vitrina & & 6,79 & 6,72 \\
\hline 13 & Vitrina & & 6,76 & 6,52 \\
\hline 14 & Vitrina & & 6,83 & 6,78 \\
\hline 15 & Vitrina & & 6,59 & 6,49 \\
\hline 16 & Vitrina & & 6,75 & 6,63 \\
\hline 17 & Sala & & 7,20 & 7,10 \\
\hline 18 & Sala & & 7,32 & 7,13 \\
\hline 19 & Vitrina & & 6,70 & 6,65 \\
\hline 20 & Sala & & 6,81 & 6,71 \\
\hline
\end{tabular}

Tabla 2.- Características de las posiciones de instalación de los sensores de acidez y resultados absolutos de pH máximos y mínimos registrados 


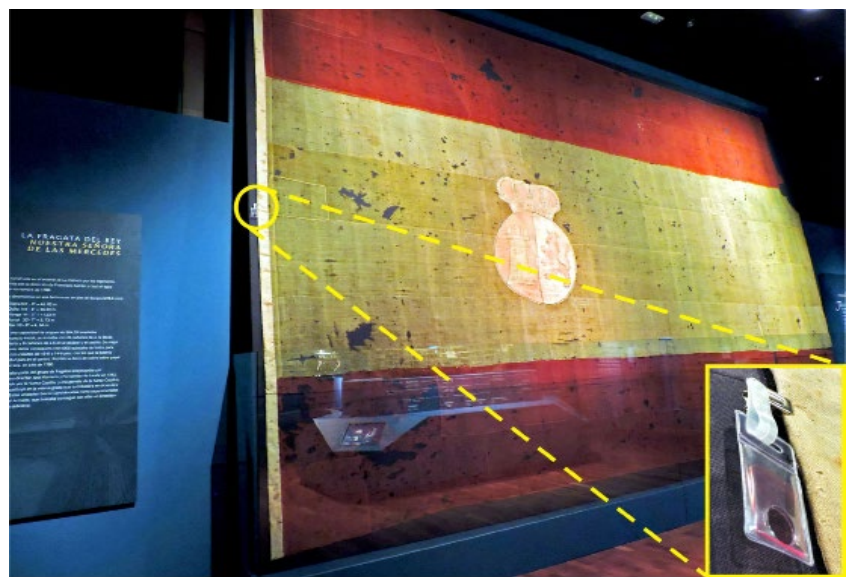

Figura 4.- Sala 6 de la exposición temporal. Posición de los sensores 3 y 11

pueden considerar, como se ha indicado anteriormente, dentro del intervalo admisible como no perjudicial para la conservación preventiva de bienes culturales, hay que señalar que se dan unas circunstancias características en esas posiciones que pueden dar cuenta de los resultados de $\mathrm{pH}$ registrados.

Por un lado el resultado máximo de $\mathrm{pH}=7,44$, registrado en ambiente de sala junto a una gran bandera extendida en un paño de la sala 6, la sala más espaciosa de la exposición temporal [figura 4], puede deberse a la zona donde hay menos contaminación de características ácidas o donde se acumula menos, ya que el volumen de aire es grande y los materiales circundantes (incluido el textil de la bandera) pueden absorber la humedad ambiental $y$, por lo tanto, amortiguar el $\mathrm{pH}$ o impedir que baje a valores ácidos. Esto se debe a que si la humedad relativa es muy baja no se formarán especies ácidas, incluso en presencia de contaminantes.

Por otro lado, el resultado mínimo de $\mathrm{pH}=6,49$, registrado en el interior de la vitrina que contiene monedas de plata y de oro rescatadas del fondo marino en donde se encontraba

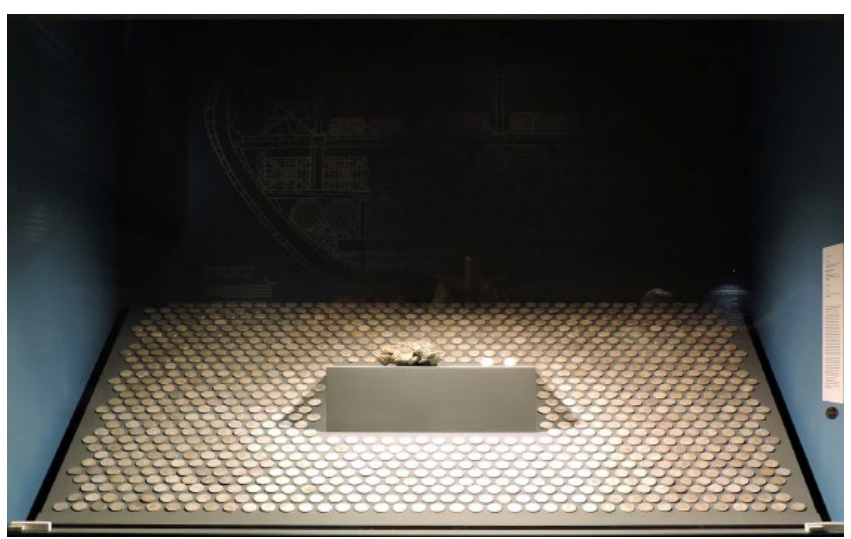

Figura 5.- Vitrina de la sala 6 de la exposición temporal que contiene monedas en la que se ubicó el sensor 15 el pecio [figura 5], se puede explicar por el volumen limitado de aire de la vitrina y por su contenido, ya que no puede descartarse que las monedas se limpiaran tras su rescate con procedimientos agresivos, como desincrustantes y decapantes que dejaran residuos de tipo ácido en la superficie de las monedas y que se desorben lentamente produciendo emisiones que permanecen en el volumen de aire disponible en el interior de la vitrina. Aunque la vitrina no está herméticamente cerrada, su ventilación es limitada y probablemente es insuficiente para intercambiar con el ambiente de sala todas las emisiones que se producen en su interior. Hay que señalar a este respecto que la hoja de vidrio frontal de esta vitrina no se retiró por razones de seguridad durante la instalación y medidas con los sensores, y que estos se introdujeron en la vitrina a través de una ranura lateral de ventilación de unos pocos milímetros de apertura. Esto quiere decir, además, que la vitrina de las monedas mantuvo sus características ambientales durante las evaluaciones llevadas a cabo, a diferencia de las otras vitrinas en las que se instalaron sensores, ya que los vidrios se retiraron momentáneamente para introducir y sacar los sensores, dando lugar a una cierta ventilación adicional del espacio interior de la vitrina.

La diferencia entre los valores máximo y mínimo de $\mathrm{pH}$ registrados (parámetro $\Delta \mathrm{pH}$ ) en una determinada posición durante el tiempo total de evaluación es muy útil a la hora de tasar los cambios que experimenta la acidez ambiental en un entorno concreto, y que afectarán directamente a las condiciones particulares de conservación de los bienes culturales de las inmediaciones. El significado de $\Delta \mathrm{pH}$ hace referencia a la estabilidad ambiental y al riesgo de choque ácido, que es el origen de la degradación química de la mayoría de los materiales.

La degradación de cualquier material es la consecuencia directa de una serie de reacciones químicas cuya velocidad aumentacuandoseproducendesviaciones delaneutralidad. Esto quiere decir que tanto los iones $\mathrm{H}^{+}$(ácidos) como los $\mathrm{OH}^{-}$(básicos) actúan o bien como reactivos en las reacciones de degradación química, o bien como catalizadores que las aceleran. De hecho los ambientes no neutros (ácidos o básicos) junto con los efectos de las radiaciones luminosas son las causas más severas y frecuentes del deterioro de los bienes culturales. Los materiales presentes en los bienes culturales de la exposición objeto de este trabajo se pueden agrupar en estos tipos: metales, textiles, papeles, maderas y cerámicas. Solo las cerámicas presentan buena resistencia quimocofísica frente a los agentes atmosféricos comunes que causan degradación. Los metales son los más expuestos a la corrosión química y electroquímica, y en ellos se producen reacciones muy agresivas de oxidación y sulfuración al entrar en contacto con los contaminantes y la humedad, que dan como resultado la formación de costras de corrosión y pérdidas masivas de material. Los textiles son muy sensibles a los ácidos, ya que sus fibras orgánicas naturales (lino, algodón, seda, celulosa, etc.) se oxidan y se hidrolizan, lo que provoca su destrucción física. Algo semejante sucede con el papel y afines, ya sea 
de fabricación tradicional con materiales naturales (papiro, pergamino, arroz, cáñamo, yute, lino, algodón, seda, etc.) o de tipo contemporáneo a base de derivados de la celulosa (a partir de 1830). En el primer caso, los materiales tienen una reserva natural alcalina que los protege hasta cierto límite de los contaminantes ácidos; mientras que en el segundo caso las fibras vegetales de la celulosa se van fragilizando progresivamente debido a la hidrólisis favorecida por las emisiones y medios ácidos (Bueno Vargas y Vázquez Jiménez 2011). Las maderas se degradan sensiblemente si el medio en que se conservan se aparta de la neutralidad. En presencia de bases la lignina y la hemicelulosa se disuelven, y la presencia de ácidos afecta a la celulosa e hidroliza sus fibras, especialmente si la humedad relativa es elevada.

Aunque no hay estándares de $\mathrm{pH}$ ambiental, se considera que el intervalo neto de $\mathrm{pH}$ admisible para la adecuada conservación de los bienes culturales se sitúa entre 6,5 y 7,5. Por otro lado, aunque no existen recomendaciones avaladas sobre los valores máximos admisibles o recomendables de $\Delta \mathrm{pH}$, se ha considerado como límite el valor de $\Delta \mathrm{pH}=$ 0,8 (Peña Poza 2014: 83). Es decir, las oscilaciones de pH menores de 0,8 para una misma posición o entorno se consideran dentro del umbral razonable por debajo del cual no existe riesgo de choque ácido. No obstante, los valores de $\Delta \mathrm{pH}$ deben interpretarse conjuntamente con los valores promedio de $\mathrm{pH}$ [figura 3], ya que estos últimos revisten aún más importancia que los de $\Delta \mathrm{pH}$ desde el punto de vista de la conservación neta de los bienes culturales. Según este criterio las posiciones donde se instalaron los sensores se pueden clasificar en función de su riesgo de choque ácido, de modo que se considera como parámetro prioritario el valor promedio de $\mathrm{pH}$ y como secundario el valor correspondiente de $\Delta \mathrm{pH}$. Se pueden dar cuatro combinaciones distintas de valores promedio de $\mathrm{pH}$ y de $\Delta \mathrm{pH}$ que se priorizan en cuanto a riesgo de choque ácido como: bajo ( $\mathrm{pH}$ promedio $>6,5$ y $\Delta \mathrm{pH}<0,8)$; leve $(\mathrm{pH}$ promedio $>6,5$ y $\Delta \mathrm{pH}>0,8)$; medio ( $\mathrm{pH}$ promedio $<6,5$ y $\Delta \mathrm{pH}<0,8)$ y alto ( $\mathrm{pH}$ promedio $<6,5$ y $\Delta \mathrm{pH}>0,8)$.

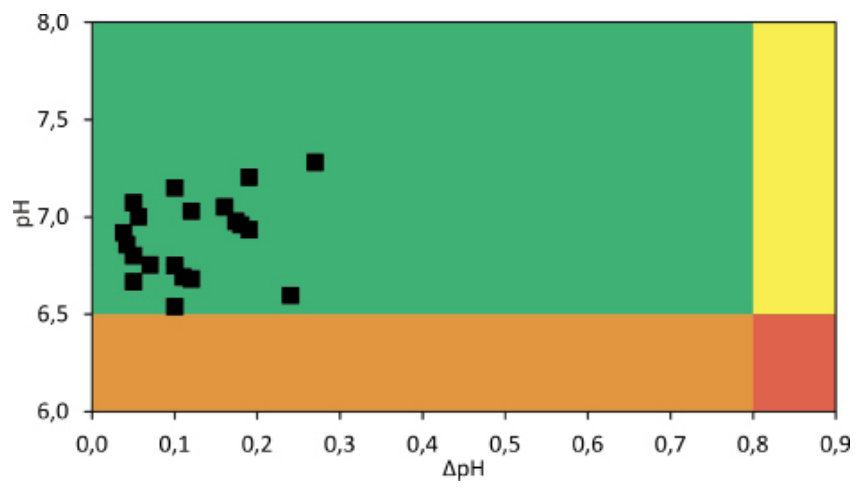

Figura 6.- Representación gráfica de los valores promedio de $\mathrm{pH}$ en función de sus correspondientes valores de $\Delta \mathrm{pH}$, para cada posición de los sensores instalados. Cada color indica un nivel de riesgo de choque ácido distinto (verde = bajo; amarillo = leve; naranja $=$ medio; rojo $=$ alto)
La figura 6 muestra el riesgo de choque ácido de cada posición evaluada basado en los resultados del parámetro $\Delta \mathrm{pH}$. Puede observarse que todas las posiciones evaluadas se sitúan en la zona de riesgo bajo de choque ácido (zona verde). Por lo tanto el ambiente en la exposición temporal, dentro y fuera de vitrinas, no supone peligro para la conservación preventiva de los bienes culturales que alberga. Es interesante señalar que en la figura 6 los datos más alejados del conjunto corresponden, por una parte, al de la posición 11 junto a la gran bandera en ambiente de la sala 6; y, por otra parte, a la posición 13 en una vitrina que contiene objetos de metal y madera, y a la posición 15 de la vitrina que contiene monedas [tabla 2]. Las posiciones 11 y 13 son las que presentan más oscilaciones de $\mathrm{pH}(\Delta \mathrm{pH} 0,27$ y 0,24 , respectivamente) y son, por lo tanto, las de menor estabilidad, aunque los resultados cuantitativos de $\mathrm{pH}$ promedio y de $\Delta \mathrm{pH}$ son aceptables de cara a la conservación preventiva. En cuanto a la posición 15 , es la que presenta el valor promedio de $\mathrm{pH}$ más ácido, como ya se ha indicado, aunque sus oscilaciones de $\mathrm{pH}$ son pequeñas $(\Delta \mathrm{pH}=0,10)$. Estos resultados se explican porque la vitrina de las monedas permaneció rigurosamente cerrada durante todo el periodo de la exposición temporal, incluso para la instalación de los sensores y durante las operaciones de toma de resultados de los mismos. Consecuentemente el intercambio de aire con el ambiente de sala se limitó exclusivamente a la pequeña ventilación que permiten las ranuras laterales de la hoja de vidrio frontal de dicha vitrina. El resultado es un ambiente muy estable, con pocas oscilaciones de $\mathrm{pH}$, pero con un grado incipiente de acidez $(\mathrm{pH}$ promedio de $6,54)$, producido por los objetos que contiene, como se ha indicado más arriba.

Los resultados de riesgo de choque ácido en el espacio expositivo de la exposición temporal deben, asimismo, compararse con los resultados que se obtuvieron mediante evaluaciones anteriores en la exposición permanente y otros espacios del Museo Naval, en las que se instalaron varios sensores en el exterior de diferentes fachadas del edificio (Peña-Poza et al. 2014). En la mayoría de dichas posiciones de exterior los valores de $\Delta \mathrm{pH}$ estaban comprendidos entre 1,8 y 2,9, aproximadamente. Estos resultados indican que el ambiente exterior del Museo Naval (Paseo del Prado muy próximo a la Plaza de Cibeles de Madrid) supone un riesgo de choque ácido alto y que una ventilación no controlada con aire del exterior puede ser peligrosa o perjudicial a efectos de la calidad del aire y la conservación preventiva. Sin embargo, a la vista de los resultados de riesgo de choque ácido registrados en los diferentes espacios expositivos de la exposición temporal de este estudio (riesgo bajo), se puede decir que la estrategia de ventilación y mantenimiento de la calidad del aire por parte del Museo Naval es muy adecuada y favorable para promover la conservación de los bienes expuestos ya que, a pesar de contar únicamente con filtro de partículas, no se produce la entrada de especies ácidas debidas a la contaminación existente en el exterior del edificio. 
Las posiciones que se seleccionaron en la evaluación llevada a cabo en julio de 2014 [tabla 1, posiciones 1 a 4] se seleccionaron también en la evaluación de octubre de 2104, con la intención de establecer, en su caso, similitudes o diferencias en la respuesta de los sensores en periodos cálido y frío. Las posiciones equivalentes fueron las siguientes: 1 y 17; 2 y 10; 3 y 11; 4 y 9. Las diferencias máximas de los $\mathrm{pH}$ registrados en posiciones equivalentes en periodos cálido y frío no superaron 0,3 unidades de la escala de $\mathrm{pH}$. Esta pequeña diferencia puede deberse a leves oscilaciones de la temperatura y la humedad relativa, así como al número neto de visitantes y su impacto en el ambiente local de la exposición temporal que, por otro lado, está climatizada. En cualquier caso estas variaciones de $\mathrm{pH}$ en posiciones equivalentes en periodos cálido y frío no suponen una amenaza para la conservación preventiva de los bienes de la exposición.

Por último se analiza la evolución de las respuestas de los sensores con el tiempo de exposición [figura 7].

La evolución del sensor 9 [tabla 1 y figura 2] representa el comportamiento característico de un sensor en una posición estable, ya que el pH varía muy poco durante todo el periodo de evaluación, y dichas variaciones se producen en todo caso en un intervalo de $\mathrm{pH}$ alrededor de la neutralidad $(\mathrm{pH}=7)$. Se trata de una posición de ambiente de sala en un espacio donde se reproduce un audiovisual sobre el combate llevado a cabo en el Cabo Sta. María entre la flota española y la británica, durante el cual la fragata Ntra. Sra. de las Mercedes fue hundida. La evolución del sensor 10 [tabla 1 y figura 2] se corresponde con otra posición estable en la que el $\mathrm{pH}$ varía muy poco, y cuyas oscilaciones se producen alrededor de la neutralidad. Este sensor se instaló en el interior de un modelo de madera de la fragata colocado (sin vitrina) en el centro de la sala 6. A partir de los datos registrados se puede afirmar que en esta posición el pH no indica la presencia de ningún tipo de sustancia ácida que pudieran emitir las maderas con las que se construyó el modelo. También hay que tener en cuenta que según indicó el maestro del Museo Naval que realizó el modelo, se utilizaron maderas nobles originales envejecidas y de la misma procedencia que las usadas en la fragata Mercedes. Por lo tanto, no se espera que dichas maderas puedan emitir sustancias de naturaleza ácida dado su largo periodo de envejecimiento.

La evolución del sensor 19 [tabla 1 y figura 2] es característica de una posición que, partiendo de un $\mathrm{pH}$ inicial próximo al neutro, experimenta una disminución debido a que el ambiente es ligeramente ácido y a continuación se estabiliza a dicho $\mathrm{pH}$ a lo largo del resto del periodo de evaluación. Esta posición (sensor número 19) corresponde a una vitrina relativamente pequeña que contiene una vasija de cerámica y naipes del siglo XIX, pudiendo estos últimos emitir sustancias ácidas debido a su propia naturaleza. Es conocido que el papel de esa época proporciona un entorno ligeramente ácido (Peña-Poza et al. 2011 b). La evolución del sensor 15 [tabla 1 y figura 2]

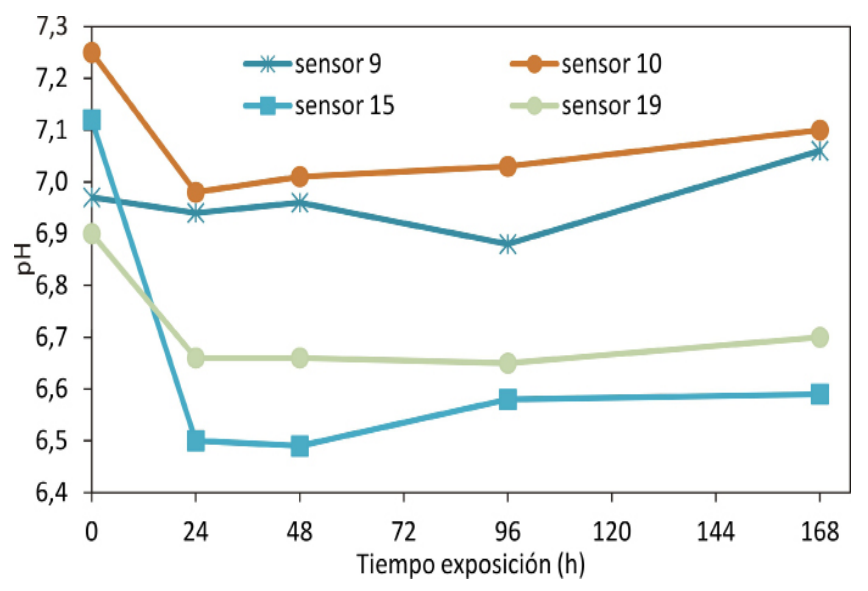

Figura 7.- Evolución del pH registrado por algunos de los sensores instalados en función del tiempo de exposición

representa la posición más crítica de este estudio: partiendo de un $\mathrm{pH}$ inicial prácticamente neutro experimenta una disminución abrupta como consecuencia del pH ácido del interior de la vitrina y posteriormente oscila entre valores semejantes de $\mathrm{pH}$ que confirman el ambiente ácido dentro de la vitrina. Este comportamiento del sensor 15 está asociado a las características de las monedas ubicadas en su interior que, como se ha explicado anteriormente, pudieron ser tratadas con diversas sustancias químicas o limpiadores más o menos agresivos que favorecen o bien las emisiones ácidas, o bien que dejaron residuos que se desorben posterior y lentamente.

\section{Conclusiones}

La utilización de un protocolo de seguimiento y control de la acidez ambiental que emplea sensores químicos de respuesta óptica ha permitido obtener información acerca de las condiciones de conservación, tanto en el ambiente de las salas como en el interior de las vitrinas, en la exposición temporal El último viaje de la fragata Mercedes. La razón frente al expolio, que tuvo lugar en el Museo Naval de Madrid entre junio de 2014 y enero de 2015 . Un estudio de conservación preventiva de estas características es la primera vez que se ha llevado a cabo en una exposición temporal.

Los resultados de $\mathrm{pH}$ ambiental obtenidos han demostrado que tanto en el ambiente libre de las salas (espacios expositivos) como en el interior de las vitrinas, el riesgo de choque ácido es bajo. En algunas vitrinas se ha detectado un ligero ambiente ácido relacionado con los objetos expuestos y, más concretamente, con los posibles tratamientos previos a los que fueron sometidos. En cualquier caso, las estrategias de ventilación general, ventilación limitada de las vitrinas y control ambiental del espacio expositivo en todo su conjunto son adecuadas para la correcta conservación preventiva de los bienes culturales expuestos. La instalación de los sensores y los datos obtenidos en las distintas posiciones han supuesto 
un control pasivo de la acidez ambiental en la muestra temporal, lo cual ha resultado beneficioso tanto para la institución prestataria (Museo Naval de Madrid) como para las diversas instituciones prestadoras. Los sensores han aportado, en general, valores promedio de $\mathrm{pH}$ muy próximos a la neutralidad teórica, lo que hace innecesario llevar a cabo un control activo que vaya más allá de las estrategias de ventilación habituales. Los materiales reaccionan con el tiempo y ello depende de la reactividad del medio. Si se comprueba que el medio no tiene especies ácidas y su humedad relativa y temperatura son equilibradas, como ocurre en este caso, la reactividad resultante será muy baja o casi nula y, por tanto, no sería necesario utilizar un medio activo de control ya que, además, los valores de pH están próximos a la neutralidad.

En consecuencia y a partir de los datos proporcionados por este estudio, el Museo Naval ha podido constatar que las pinturas, barnices, adhesivos y restantes materiales presentes en las salas destinadas a exposiciones temporales, que se hallan sujetos a la Directiva 1999/13/CE, no han provocado niveles de pH nocivos para las colecciones expuestas, así como que el filtrado de contaminantes del aire es adecuado. Toda esta información ha comenzado a incluirse en los informes sobre las instalaciones que el Museo Naval prepara y facilita habitualmente a las instituciones prestadoras que colaboran en la realización de exposiciones temporales. Asimismo, la información sobre los ligeros valores ácidos relacionados con la vitrina en la que se exponían las monedas, ha servido para advertir a la institución que custodia estos materiales de los valores registrados, circunstancia que ha promovido el inicio de acciones de investigación para paliar estos efectos.

\section{Agradecimientos}

Los autores agradecen a Fernando Agua y Juan Félix Conde (Instituto de Historia, CSIC) su inestimable ayuda y colaboración en la realización del estudio. También desean expresar su agradecimiento a los proyectos ref. HAR-201230769 (Ministerio de Economía y Competitividad) y S2013/ MIT-2914 (Comunidad de Madrid y fondos estructurales UE). Asimismo agradecen a la red TechnoHeritage de Ciencia y Tecnología para la Conservación del Patrimonio Cultural su apoyo profesional.

\section{Bibliografía}

BAMBERGER, J.A., HOWE, E.G., WHEELER, G. (1999). “A variant Oddy test procedure for evaluating materials used in storage and display cases". Studies in Conservation, 44: 86-90.

BUENO VARGAS, J., VÁZQUEZ JIMÉNEZ, E. (2011). "Archivos municipales en pequeñas y medianas poblaciones: principales materiales y pautas básicas para la conservación de sus fondos". Arch-e. Revista Andaluza de Archivos, 4: 1-25.
CAMUFFO, D., VAN GRIEKEN, R., BUSSE, H.J., STURARO, G., VALENTINO, A., BERNARDI, A., BLADES, N., SHOOTER, D., GYSELS, K., DEUTSCH, F., WIESER, M., KIM, O., ULRYCH, U. (2001). "Environmental monitoring in four European museums", Atmospheric Environment, 35 Suppl. 1: 127-140.

FERNÁNDEZ, C. (coord.) (2008). Conservación preventiva y procedimientos en Exposiciones Temporales. Madrid: GEIIC y Fundación Duques de Soria.

FORNIÉS MATÍAS, Z. (2011). La climatización de los depósitos de archivos, bibliotecas y museos como método de conservación. Gijón: Ediciones Trea, Serie Conservación y Restauración del Patrimonio $n^{\circ} 3$.

FORNIÉS MATÍAS, Z. (2012). "Criterios básicos para una evaluación climática de calidad". En Actas del V Congreso del Grupo Español del GEIIC. Patrimonio Cultural, criterios de calidad en intervenciones, Madrid, 307-314.

GALL ORTLIK, A. (2004). "El test de Oddy: un instrumento de selección de materiales aptos para la conservación-restauración", Unicum, 3: 100-103.

GARCÍA FERNÁNDEZ, I. M. (2014). "Historia de la conservación preventiva. Parte II", Ge-conservación, 6: 5-18.

LLORENTE, A., PEÑA-POZA, J., DOMÍNGUEZ, J.F., GIL, C., GARCÍAHERAS, M., VILLEGAS, M.A. (2013). "Evaluation of environmental conditions of the Museo del Ejército (Toledo, Spain) by means of Sol-Gel optical sensors". En Science and Technology for the Conservation of Cultural Heritage, Rogerio-Candelera, M.A., Lazzari, M., Cano, E. (ed.). London: Taylor \& Francis Group, 15-19.

MICHALSKI, S. (1998). "Climate control priorities and solutions for collections in historic buildings", Historic Preservation Forum, 12 (4): 8-14.

MUÑOZ-CAMPOS, P. (2006). “La conservación preventiva en los museos de artes decorativas. El reto del almacenamiento", Revista de Museología, 36: 124-134.

ODDY, W.A. (1973). "An unsuspected danger in display", Museums Journal, 73: 27-28.

PEÑA-POZA, J., GARCÍA-HERAS, M., PALOMAR, T., LAUDY, A., MODZELEWSKA, E., VILLEGAS, M.A. (2011 a). "Environmental evaluation with chemical sensors in the palace museum of Wilanów (Warsaw, Poland)", Bulletin of the Polish Academy of Sciences-Technical Sciences, 59 (3): 247-252.

PEÑA-POZA, J., CONDE, J.F., PALOMAR, T., AGUA, F., GARCÍAHERAS, M., VILLEGAS, M.A. (2011 b). "Environmental evaluation of the holdings at the CCHS-CSIC Tomas Navarro Tomas Library", Revista Española de Documentación Científica, 34(1): 65-78.

PEÑA POZA, J. (2014). Optimización, comportamiento y preindustrialización de sensores ambientales basados en la tecnología Sol-gel. Tesis doctoral, Madrid: Universidad Autónoma de Madrid < http://hdl.handle.net/10486/664117>. 
PEÑA-POZA, J., AGUA, F., CONDE, J.F., DE SAN PÍO, P., GARCÍA RAMÍREZ, S., GÁLVEZ FARFÁN, J.M., MORENO MARTÍN, J.M., GONZÁLEZ RODRIGO, M., GARCÍA-HERAS, M., VILLEGAS, M.A. (2014). "Air quality assessment and protection treatments impact on the collection of the Museo Naval (Madrid, Spain)." En Science, Technology and Cultural Heritage, Rogerio-Candelera, M.A. (ed.). London: Taylor \& Francis Group, 47-52.

PNCP (2011). Plan Nacional de Conservación Preventiva. Madrid: Ministerio de Cultura, Secretaría de Estado de Cultura.

THOMSON, G. (2008). The Museum Environment. 2nd ed. Oxford: Butterworth-Heinemann.
VV.AA. (2000). Towards a European Preventive Conservation Strategy Adopted at the Vantaa Meeting. Helsinki: ICCROM (International Centre for the Study of the Conservation and Restoration of Cultural Property) and Institute of Art and Design.

VV.AA. (2006). Exposiciones temporales: organización, gestión y coordinación. Madrid: Subdirección General de Promoción de las Bellas Artes, Ministerio de Cultura.

VILLEGAS BRONCANO, M.A., GARCÍA HERAS, M., PEÑA POZA, J., DE ARCAS CASTRO, G., BARRERA LÓPEZ DE TURISO, E., LÓPEZ NAVARRO, J.M., LLORENTE ALONSO, A. (2010). Sistema para la determinación de acidez ambiental y método que hace uso del mismo. Patente española de invención ref. P201031071.

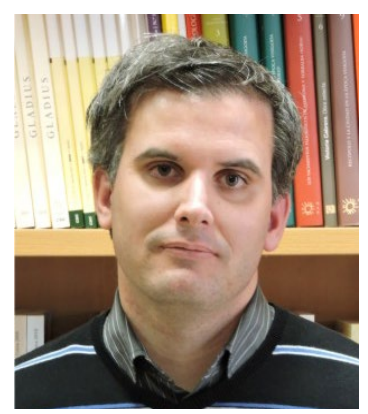

\author{
Javier Peña Poza \\ Instituto de Historia, CCHS-CSIC. Madrid \\ javier.pena@cchs.csic.es
}

Doctor en Ciencias Químicas por la Universidad Autónoma de Madrid (2014). Entre 2009 y 2013 fue investigador contratado en el Instituto de Historia (CCHS-CSIC, Madrid) con cargo al Programa Consolider Ingenio TCP-CSD2007-00058 y en marzo de 2015 se ha reincorporado de nuevo al Instituto de Historia con otro contrato con cargo al programa Geomateriales2-CM S2013/MIT-2914. Sus líneas de investigación se centran en la aplicación de técnicas físico-químicas de caracterización a materiales inorgánicos y al desarrollo de estrategias de conservación preventiva a través de la tecnología de sensores ambientales.

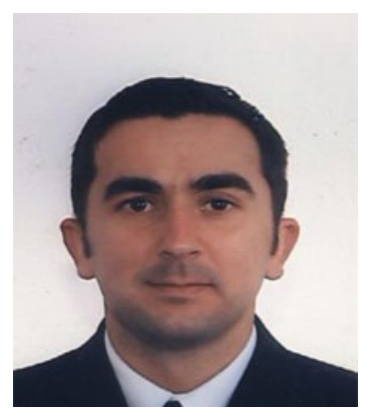

\title{
José María Gálvez Farfán
}

Museo Naval, Ministerio de Defensa. Madrid

jgalfarn@fn.mde.es

Licenciado en Bellas Artes en la especialidad de restauración de escultura por la Universidad de Granada (2000). Entre 2000 y 2002 realizó trabajos de restauración arqueológica en Málaga. Desde 2002 es Oficial de la Armada, Teniente de Navío, Ministerio de Defensa, destinado en el Museo Naval de Madrid desde 2003, en donde es restaurador del Departamento de pintura y escultura. Actualmente es el responsable del Departamento de Conservación Preventiva. 


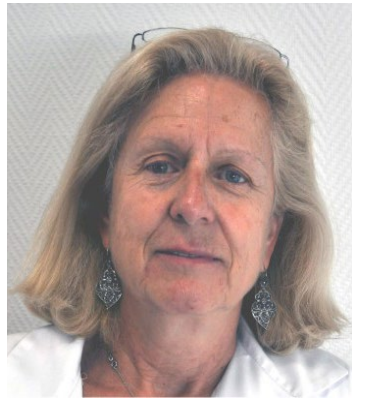

\title{
Mercedes González Rodrigo
}

Museo Naval, Ministerio de Defensa. Madrid

mgonr11@fn.mde.es

Titulada en Restauración de documento gráfico por el Centro Nacional de Conservación y Microfilmación Documental y Bibliográfica del Ministerio de Cultura (1977). Es también graduada en Conservación y Restauración de Documento Gráfico por la Escuela de Artes Aplicadas de Madrid (1982). Desde 1983 es Conservadora y Restauradora de Documento Gráfico en el Museo Naval de Madrid.

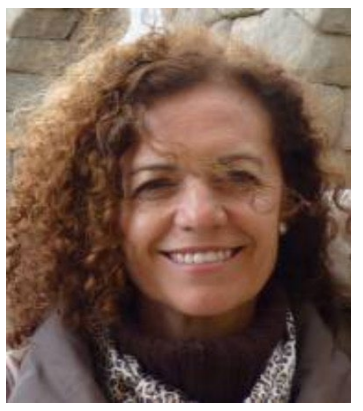

\section{Susana García Ramírez}

Museo Naval, Ministerio de Defensa. Madrid

sgarram1@et.mde.es

Licenciada con grado en Geografía e Historia, especialidad de Historia Antigua, por la Universidad Complutense de Madrid. Ha dirigido su trayectoria científica a la Arqueología, la Documentación y la Museología. En esta última, ejercida en el Ayuntamiento de Madrid, la Universidad Autónoma de Madrid, el Ministerio de Cultura y el Ministerio de Defensa, ha trabajado en diversos aspectos de la Conservación referida especialmente a criterios de intervención en textiles (Museo del Ejército, Jefatura del Departamento de Uniformidad y Simbología, 2007-2012) y a control de factores ambientales (Museo Naval, Jefatura del Departamento de Conservación Preventiva y Restauración, 2012-2014). Ha comisariado las exposiciones temporales A la cabeza del Ejército (2012) y El último viaje de la fragata Mercedes. La razón frente al expolio (2014). En la actualidad es conservadora jefe del Área de Investigación del Museo Naval.

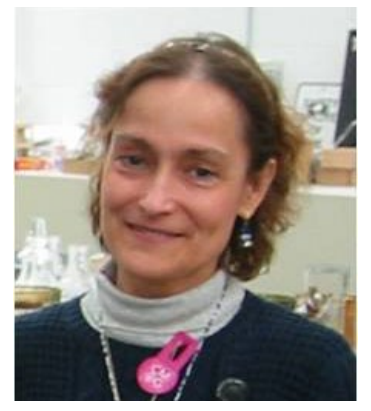

\author{
María Ángeles Villegas Broncano \\ Instituto de Historia, CCHS-CSIC. Madrid \\ mariangeles.villegas@cchs.csic.es
}

Doctora en Ciencias Químicas por la Universidad Autónoma de Madrid (1987) e Investigadora Científica del CSIC en el Instituto de Cerámica y Vidrio (1984-2001), Centro Nacional de Investigaciones Metalúrgicas (2001-2007) e Instituto de Historia (desde 2007). Es la responsable de un grupo de investigación sobre materiales del Patrimonio Cultural y directora del curso de postgrado "Gestión de recursos técnicos en la conservación del patrimonio cultural". Sus líneas de investigación son: Conservación integral (curativa y preventiva) y protección del Patrimonio Histórico y Cultural; Sensores ambientales y sistemas avanzados de conservación preventiva; Arqueometría de materiales (vidrio, cerámica, metales, aleaciones, capas pictóricas, etc.). 


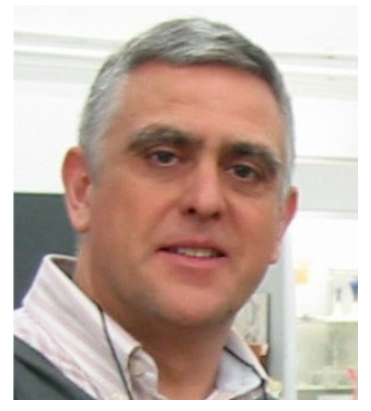

\section{Manuel García Heras}

Instituto de Historia, CCHS-CSIC

manuel.gheras@cchs.csic.es

Doctor en Historia con Premio Extraordinario por la Universidad Complutense de Madrid (1997). Entre 1999 y 2001 fue investigador postdoctoral Fulbright en la Smithsonian Institution, Washington D.C., EE.UU.; y entre 2002 y 2005 investigador postdoctoral del programa I3P en el Centro Nacional de Investigaciones Metalúrgicas (CENIM-CSIC). Desde 2005 es Científico Titular en el Instituto de Historia (CCHS-CSIC), Madrid. Sus líneas de investigación se centran en la interacción entre ciencias experimentales e historia, combinando una visión histórica y científica en la aproximación a cuestiones de tecnología y conservación de materiales antiguos. Estas líneas se llevan a cabo principalmente en los campos de la Arqueometría y la conservación del Patrimonio Cultural 